\title{
Perinatal Depression: Challenges and Opportunities
}

\author{
Rada K. Dagher, PhD, MPH, ${ }^{1}$ Hannah E. Bruckheim, ${ }^{2}$ Lisa J. Colpe, PhD, MPH, ${ }^{3}$ \\ Emmeline Edwards, $\mathrm{PhD},{ }^{4}$ and Della B. White, $\mathrm{PhD}^{4}$
}

\begin{abstract}
Perinatal depression (PND) is a major depressive episode during pregnancy or within 4 weeks after childbirth up to a year. Risk factors for PND include stressful life events, history of depression, poor social support, unplanned and unwanted pregnancies, poor relationship quality, current or previous abuse, and low socioeconomic status. This mental disorder has been shown to have negative effects on mothers' quality of life and their intimate relationships, birth outcomes, and breastfeeding likelihood, as well as long-term effects on children's cognitive and emotional development. To date, no nationally representative study has examined whether there are socioeconomic and/or racial/ethnic differences in PND. This study discusses the prevalence and risk factors for PND, as well as its health consequences for mothers and children, the reasons for its underreporting and undertreatment, the evidence for different screening instruments and different treatment options, and the existing supportive policies to address this disorder in the United States. We conclude with outlining next steps in addressing the gaps in the literature on PND.
\end{abstract}

Keywords: perinatal depression, racial/ethnic differences, underreporting/undertreatment, supportive policies

\section{Introduction}

$\mathbf{T}$ HE PERINATAL PERIOD, an important transition to motherhood, represents a vulnerable time for developing mental health disorders, including perinatal depression (PND). ${ }^{1}$ PND is defined in the Diagnostic and Statistical Manual of Mental Disorders-5th edition (DSM-5) as the occurrence of a major depressive episode during pregnancy [antenatal depression (AND)] or following childbirth [postpartum depression (PPD)], with use of the "with peripartum onset" specifier for depressive disorders (onset during pregnancy or within 4 weeks after delivery). ${ }^{2}$ PND should not be confused with the "postpartum blues," which is a commonly experienced state of fluctuating mood, fatigue, tearfulness, irritability, and feelings of anxiety that generally resolves within the 10-14 days following delivery. ${ }^{1}$

Although biological factors may influence mood in the early postpartum recovery period, growing evidence indicates that an accumulation of psychological stressors in the 1st year after delivery also contributes to the onset or recurrence of depressive episodes. ${ }^{3}$ Symptoms of depressive disorders include depressed mood, loss of energy and/or interest in daily activities, sleep problems or appetite/weight changes, poor concentration, feelings of worthlessness, guilt and/or hopelessness, and suicidal thoughts. For a major depression diagnosis, five or more specific symptoms must be present for at least 2 weeks, one of which must be depressed mood or loss of interest. ${ }^{2}$ By strict DSM-5 standards, depressive episodes that occur beyond the 4-week period after delivery are classified as a depressive disorder without the peripartum modifier. In practice, however, the prenatal period and up to 1 year postpartum is a risk period that is both critical to recognize and amenable to depression screening and treatment. ${ }^{4}$

\section{Prevalence of Depression in the Perinatal Period}

Depression is one of the most common serious complications of pregnancy. According to a 2005 national review, the

\footnotetext{
${ }^{1}$ Division of Scientific Programs, National Institute on Minority Health and Health Disparities, National Institutes of Health, Bethesda, Maryland, USA.

${ }^{2}$ National Heart, Lung, and Blood Institute, Bethesda, Maryland, USA.

${ }^{3}$ National Institute of Mental Health, National Institutes of Health, Bethesda, Maryland, USA.

${ }^{4}$ Division of Extramural Research, National Center for Complementary and Integrative Health, National Institutes of Health, Bethesda, Maryland, USA.

All co-authors contributed equally to the article and are listed in alphabetical order.

(C) Rada K. Dagher et al. 2020; Published by Mary Ann Liebert, Inc. This Open Access article is distributed under the terms of the Creative Commons Attribution Noncommercial License (http://creativecommons.org/licenses/by-nc/4.0/) which permits any noncommercial use, distribution, and reproduction in any medium, provided the original author(s) and the source are cited.
} 
prevalence of major and minor depression in the United States is $8.5 \%-11 \%$ during pregnancy and 6.5\%-12.9\% during the first postpartum year. ${ }^{5}$ A 2016 review that focused on longitudinal studies reported an average prevalence rate of $17 \%$ for AND and $13 \%$ for PPD; moreover, $39 \%$ of women who experienced AND went on to develop PPD. ${ }^{6}$

Some research evidence points to racial/ethnic differences in PND prevalence rates in the United States. A systematic review of racial/ethnic disparities in AND found that the overall prevalence was $10 \%-30 \%$ and was higher among African American and Hispanic women compared to white women. ${ }^{7}$ However, this review compared studies that used different designs, instruments, and settings, which limited generalizability. An analysis of 2018 data from the Pregnancy Risk Assessment Monitoring System found that the prevalence of self-reported PPD symptoms averaged $13.2 \%$ and was higher among American Indian/Alaska Native (22.0\%), Asian/Pacific Islander (19.2\%), and black women (18.2\%) than among white women $(11.4 \%) .{ }^{8}$ However, PPD symptoms were measured with the Patient Health Questionnaire-2 (PHQ-9) instrument, AND symptoms were not measured, and only 31 states were included. Studies also point to increased PND prevalence among low-income women. A recent review of the literature revealed mixed findings, in which some studies reported that up to $50 \%$ of low-income women experienced PND, while other studies that focused on women who received public assistance or used public-sector services reported 12-month depression rates of $12 \%-36 \%$. $^{9}$ None of these studies is nationally representative and they differ in designs, instruments, and settings; thus, any evidence regarding racial/ethnic and income differences in PND rates is inconclusive.

The coronavirus disease 2019 (COVID-19) pandemic caused by the novel coronavirus (SARS-CoV-2) is another factor to consider. Recently published data from China on the mental health impact of the COVID-19 outbreak show increased rates of antenatal ${ }^{10}$ and postpartum depressive symptoms among Chinese women. ${ }^{11}$ Similarly, a convenience sample of mothers in the United States of children 0-18 months of age showed elevated rates of depressive $(34.1 \%)$ and anxiety $(34.6 \%)$ symptoms. ${ }^{12}$ Thus, reassessment of PND prevalence rates and examination of immediate and long-term effects of the pandemic on these rates could be potential avenues for future research.

The aforementioned prevalence statistics mainly relied on self-reported depressive symptoms using different screening tools and sampling techniques and do not constitute nationally representative estimates of medical diagnoses of PND. A study of depression diagnoses during delivery hospitalizations that analyzed data from the National Inpatient Sample and 31 State Inpatient Databases found that depression rates were 28.7 per $1,000(2.87 \%)$ in $2015 .^{13}$ This showed a discrepancy between estimates of diagnosis rates and self-reported rates. Future research efforts could be geared toward examining the reasons behind this discrepancy and whether differences in PND diagnoses by race/ethnicity and income exist.

\section{Risk Factors and Etiology of PND}

Multiple risk factors have been associated with PND, including social, psychological, biological, and genetic factors. ${ }^{4}$ Systematic reviews of risk factors for AND found the following factors to be associated with higher likelihood of AND symptoms: life stress, depression history, maternal anxiety, lack of social support, lower frequency of exercise, unintended pregnancy, Medicaid insurance, intimate partner violence, child abuse history, lower income, lower education, smoking, single status, and poor relationship quality. ${ }^{14,15}$ Recent studies also showed associations between adverse childhood experiences and AND. ${ }^{16,17}$ Moreover, obese and overweight women had a higher likelihood of elevated AND symptoms than women with normal weights. ${ }^{18}$ Notably, the literature remains more extensive on PPD risk factors than on AND.

A 2014 review of the literature and existing meta-analyses revealed that the following factors have moderate to strong associations with PPD: history of depression, AND and antenatal anxiety, neurotic personality, low self-esteem, maternity blues, stressful life events, poor marital quality, and poor social support. ${ }^{19}$ Factors with smaller associations included low socioeconomic status, single marital status, unintended pregnancy, obstetrical stressors, and an infant with a difficult temperament. ${ }^{19}$ In addition, women who developed PPD appeared to be more sensitive to the neurobiological effects of the sudden drop in estradiol levels in the first few postpartum days; moreover, data from genome-wide association studies point to the possibility that genetic variations on specific chromosomes, such as chromosome 1q21.3-q32.1, could increase predisposition to PPD symptoms. ${ }^{19,20}$ A 2020 literature review added that the strongest two risk factors for PPD are AND and current domestic abuse. ${ }^{21}$ Finally, a metaanalysis of studies from 56 countries found that the global PPD prevalence was $17.7 \%$ and that $73 \%$ of the variation across countries can be explained by higher rates of income inequality, maternal mortality, infant mortality, or women working 40 hours a week. ${ }^{22}$

To summarize, the causes of PND are likely multifactorial. Future research studies could investigate whether a geneenvironment interaction model can improve the specification of PND risk. That is, genes may provide a certain level of risk that can be exacerbated by environmental stressors, such as adverse life events, low socioeconomic status, and pregnancy complications. ${ }^{23}$ In addition, research examining whether certain factors may confer different risk levels across various racial/ethnic and income groups could enhance timely diagnosis and treatment for those at the highest risk.

\section{Health Consequences to the Mother and Offspring}

PND may present serious health consequences for both the mother and her offspring. AND has been associated with delayed fetal development, higher incidence of prematurity and low birth weight, and increased rates of such prenatal and perinatal complications as preeclampsia, placental abnormalities, and spontaneous abortion. ${ }^{15,24}$ Moreover, AND has been associated with poor maternal sleep, PPD, less exclusive breastfeeding, paternal depression, a lower Apgar score and Brazelton Neonatal Behaviour Assessment Scale at birth, infant sleep problems, perceptions of difficult infant temperament, and higher body mass index of the child, as well as a twofold increase in childhood mental disorders. ${ }^{15}$ PPD can have negative effects on maternal-infant bonding and interaction $^{25}$; adaptation to the mothering role and confidence in providing infant care ${ }^{26}$; maternal quality of life and intimate 
relationships ${ }^{25}$; children's emotional, cognitive, and behavioral development ${ }^{27}$; and the father's mental health. ${ }^{28}$ Furthermore, some perinatal women who are depressed have an increased suicide risk. Estimates have shown that suicide may account for up to $20 \%$ of maternal mortality. ${ }^{29}$

Children whose mothers suffered from PND are at increased risk for significant developmental delays, such as lower levels of activity, fewer facial expressions, lower levels of attentiveness, and physiological changes characterized by elevated cortisol levels and decreased levels of dopamine and serotonin. ${ }^{30}$ Gaps in the current literature point to a dearth of longitudinal neurodevelopmental studies of children exposed to perinatal mental illness, with or without maternal use of psychotropics during pregnancy; few longitudinal studies to determine the mediating and moderating mechanisms through which PND exerts lasting effects on the child; and scant translational research on the potential role of the microbiome in mother-infant interaction and PND. ${ }^{31-34}$

\section{Underreporting and Undertreatment of PND}

In the United States, $50 \%-70 \%$ of women with AND or PPD are undetected and undiagnosed, and nearly $85 \%$ go untreated. ${ }^{31}$ These low detection and treatment rates may be due to several factors. First, PND can be difficult to assess during the clinical encounter due to the overlap of common somatic symptoms of pregnancy or such normal experiences of motherhood as fatigue or appetite changes with symptoms of depression. ${ }^{35,36}$ Second, routine screening for PND, which helps detect more cases than clinical judgment alone, is not standard practice in the United States. ${ }^{37}$ In addition, other patient-, provider-, and system-level barriers exist to addressing and treating PND, such as mental illness stigma, cultural factors, fear of side effects of antidepressants, limited access to mental health care, limited resources and mental health training among perinatal care providers, and low levels of coordination between perinatal and mental health professionals. ${ }^{38}$

The American College of Obstetricians and Gynecologists (ACOG) has recently recommended screening women for depression and anxiety symptoms at least once during the perinatal period, coupled with appropriate initiation of treatment and referrals to behavioral therapy when indicated and having systems in place that facilitate follow-up for diagnosis and treatment. ${ }^{39}$ This recommendation came after the U.S. Preventive Services Task Force updated its 2009 routine depression screening recommendations of the general adult population to include pregnant and postpartum women, while stipulating that "screening should be implemented with adequate systems in place to ensure accurate diagnosis, effective treatment, and appropriate follow-up." 40 This was a "Rating B Recommendation," which implies high certainty that the net benefits of routine screening are moderate. ${ }^{40}$ Thus, increasing evidence supports the effectiveness of routine depression screening of women in the perinatal period.

\section{Evidence for Different Screening Instruments and Different Treatment Options}

Seven screening instruments have been validated for detecting PND during pregnancy and the postpartum period and have been deemed acceptable by the ACOG: the Edinburgh Postnatal Depression Scale (EPDS), Postpartum Depression
Screening Scale, PHQ-9, Beck Depression Inventory, Beck Depression Inventory II, Center for Epidemiologic Studies Depression Scale, and the Zung Self-Rating Depression Scale. ${ }^{39}$ However, the EPDS remains the most frequently utilized instrument in clinical and research settings for several reasons, including its brevity ( 10 items that take $<5$ minutes to complete), its exclusion of constitutional symptoms that are common during pregnancy and the postpartum period-unlike all the other instruments, and its inclusion of the anxiety symptoms that are a prominent feature of perinatal mental disorders. ${ }^{39}$ The PHQ-9 is also brief, with nine items, including a suicide item, and is utilized in obstetricgynecological settings. ${ }^{41}$ Although the aforementioned instruments have proven their reliability and validity, ${ }^{39}$ no consensus exists on the use of a standard instrument for PND screening.

Even though PND screening is important, it is not as effective without appropriate follow-up and treatment. Most studies have evaluated the effectiveness of psychological treatments for PND, such as interpersonal psychotherapy (IPT) and cognitive behavioral therapy (CBT); however, only a few studies have evaluated pharmacological treatments because pregnant and lactating women often are excluded from medication trials. In 2011, a meta-analysis of the efficacy of pharmacologic and psychological interventions found that IPT demonstrated higher effect sizes when compared to controls and was superior to CBT in PND treatment (higher effect sizes when compared to controls). The metaanalysis also showed that individual psychotherapy was superior to group psychotherapy when observing changes in symptoms from pretreatment to posttreatment. ${ }^{42}$ However, no conclusion was made regarding pharmacological treatments because there were too few studies. ${ }^{42}$ An updated 2018 meta-analysis showed that IPT was effective in reducing PND symptoms in both prevention and treatment studies. ${ }^{43}$ Another updated meta-analysis showed that CBT was effective in reducing depressive symptoms compared to control conditions in both prevention and treatment studies, and postpartum interventions were more effective than antenatal interventions in both types of studies. ${ }^{44}$ Moreover, prevention studies that had higher proportions of non-white, single, and multiparous participants showed greater reduction in depressive symptoms due to $\mathrm{CBT}^{44} \mathrm{~A}$ recent review of the reproductive safety of pharmacological interventions concluded that certain antidepressants-such as selective serotonin reuptake inhibitors, serotonin-norepinephrine reuptake inhibitors, tricyclic antidepressants, and bupropion-are deemed safe for administering to pregnant and lactating women when used appropriately. ${ }^{45}$ However, the studies cautioned that double-blind placebo-controlled studies on these antidepressants do not exist. ${ }^{45}$

\section{Complementary Health and Integrative Health Approaches for PND}

Evidence suggests that many women inquire about and prefer to use complementary health approaches during pregnancy. ${ }^{46-48}$ These include "natural products," such as dietary supplements, and mind and body approaches, such as yoga, acupuncture, and mindfulness. Reasons for use of these approaches include motivation to have greater control over childbearing experiences and concerns about the risks to their 
infants from using pharmaceuticals to treat depression. ${ }^{46,47,49}$ Indeed, pregnant women use multiple complementary health approaches and report perceptions that these approaches have some clinical benefit; however, health professionals often are not consulted before their use. ${ }^{46,47,50}$

Only preliminary evidence exists to indicate that complementary health approaches - such as folate supplementation (yet there are well-documented protective effects of folate against the development of neural birth defects ${ }^{51}$ ), yoga, and mindfulness - are safe, feasible, and acceptable for perinatal women and have the potential for improving depressive symptoms. ${ }^{48,52-55}$ These types of low-cost interventions can be important for women who have limited access to traditional treatment with psychotherapy. ${ }^{48,56}$ However, concerns have been raised about the use of these approaches for which the evidence for full efficacy and safety remains limited. ${ }^{49}$ Additional research involving more rigorous study designs with larger, diverse samples compared to more conventional psychotherapies and pharmacotherapies and research to understand the mechanisms of action of these approaches may help inform optimal dosing and efficacy of these approaches. $^{48,52,55}$

\section{Supportive Policies for PND}

Supportive policies across the United States at the state and federal government levels provide insights for addressing the undue burden that PND puts on new mothers and their families.

At the state level, there have been numerous legislative developments over the past 15 years, the first being enacted in New Jersey in $2006 .{ }^{57}$ This law mandated PND screening and education by health care providers and required doctors to ask expectant mothers about their depression history. A study that evaluated this legislation, however, showed that it had no impact on PPD screening and treatment among mothers who are Medicaid recipients. ${ }^{57}$ Illinois and West Virginia passed similar screening and education laws in 2008 and 2009, respectively. $^{58}$ Moreover, four states-Montana, Oregon, Texas, and Virginia-have passed legislation that requires mandatory education about PND for health care providers and informational materials for new parents about depressive symptoms and available resources. ${ }^{58}$ Notably, in 2012, Minnesota amended legislation to outline the eventual implementation of mandatory screening practices, which will require the Commission of Health to provide technical support to health care providers as screening becomes more widespread (Minnesota Statute $\S 145.906$ ).

At the federal government level, numerous bills have been introduced by Congress to increase federal support for PND prevention over the past 20 years. The first of these-the Melanie Blocker-Stokes PPD and Research Act, later renamed the MOTHERS Act-was first introduced in 2003 and then enacted in 2010 as part of the Affordable Care Act. ${ }^{59}$ This legislation allocated federal funds for public and nonprofit health care providers to enhance inpatient, outpatient, and home-based health and support services. The legislation also encouraged the U.S. Department of Health and Human Services to facilitate research into perinatal mood disorders and to launch a national awareness campaign. ${ }^{59}$ In addition, language from the MOTHERS Act was incorporated into the Patient Protection and Affordable Care Act Section 2952 (Support,
Education, and Research for PPD), which authorizes grants to support the establishment, operation, and delivery of effective and cost-efficient systems for providing clinical services to women with, or at risk for, PPD or psychosis.

Research in the United States and other countries has shown that access to paid leave after childbirth is associated with better perinatal mental health. ${ }^{60}$ However, the United States is the only country in the industrialized world, and one of two countries among 185 (the other is Papua New Guinea), which does not have a national paid maternity leave law. ${ }^{61}$ Thus, women's access to paid leave is contingent on their employer's policy or the state they reside in. Currently, eight states and the District of Columbia have passed some form of paid leave policy. ${ }^{62}$ Evaluation of California's Paid Family Leave program showed that before this law was passed, African American women took on average 1 week of leave after childbirth compared to 4 weeks for white women; however, several years after the leave act was implemented, both groups averaged 7 weeks of leave. ${ }^{63}$ Moreover, this law was associated with a $29 \%$ reduction in mean psychological distress symptoms, the effects being most pronounced for black, single, and low-income women. ${ }^{64}$ On December 20, 2019, the President signed a new law, the Federal Employee Paid Leave Act, which provides 2.1 million federal workers with up to 12 weeks paid leave following childbirth, adoption, or fostering.

\section{Conclusion}

PND is a debilitating mental disorder with disruptive impacts on the health of mothers, fathers, and children. Preliminary evidence shows racial and ethnic differences in PND prevalence, which could be further explored using nationally representative studies and well-validated measures for PND, including medical diagnoses. Some understudied research areas include examination of racial/ethnic disparities in accessing PND screening and treatment services. In addition, future research efforts could both devise ways to conduct safe, rigorous studies of pharmacologic treatments for mental disorders in pregnant and lactating women and carefully explore the effectiveness of complementary health approaches in comparison to conventional therapies. Finally, more studies that evaluate the impact of existing policies on the mental health of perinatal mothers, including such underserved populations as racial/ethnic minorities and lowincome women, are warranted.

\section{Acknowledgments}

We would like to acknowledge Dr. Cheryl Kassed for her helpful edits and feedback on this article.

\section{Disclaimer}

The views expressed in this article are those of the authors and do not necessarily reflect the official views of the $\mathrm{Na}$ tional Institutes of Health, the Department of Health and Human Services, or the United States federal government.

\section{Author Disclosure Statement}

The authors have no financial conflicts of interest. 


\section{Funding Information}

No funding was received for this article.

\section{References}

1. Langan R, Goodbred AJ. Identification and management of peripartum depression. Am Fam Physician 2016;93:852-858.

2. American Psychological Association. Diagnostic and statistical manual of mental disorders, 5th ed. Washington, DC: American Psychological Association, 2013.

3. Stuart-Parrigon K, Stuart S. Perinatal depression: An update and overview. Curr Psychiatry Rep 2014;16:468.

4. O'Connor EA SC, Henninger M, Gaynes BN, Coppola E, Soulsby Weyrich M. Interventions to prevent perinatal depression: A systematic evidence review for the U.S. Preventive Services Task Force Evidence Synthesis, No. 172. Rockville, MD: Agency for Healthcare Research and Quality, 2019. Available at: https://www.ncbi.nlm.nih.gov/ books/NBK537819 Accessed April 3, 2020.

5. Gaynes BN, Gavin N, Meltzer-Brody S, et al. Perinatal depression: Prevalence, screening accuracy, and screening outcomes: Summary. In: AHRQ Evidence Report Summaries. Rockville, MD: Agency for Healthcare Research and Quality, 2005.

6. Underwood L, Waldie K, D'Souza S, Peterson ER, Morton S. A review of longitudinal studies on antenatal and postnatal depression. Arch Womens Ment Health 2016;19:711-720.

7. Mukherjee S, Trepka MJ, Pierre-Victor D, Bahelah R, Avent T. Racial/ethnic disparities in antenatal depression in the United States: A systematic review. Matern Child Health J 2016;20:1780-1797.

8. Bauman B, Ko J, Cox S, et al. Vital signs: Postpartum depressive symptoms and provider discussions about perinatal depression-United States, 2018. MMWR Morb Mortal Wkly Rep 2020;69:575-581.

9. Klawetter S, McNitt C, Hoffman JA, Glaze K, Sward A, Frankel K. Perinatal depression in low-income women: A literature review and innovative screening approach. Curr Psychiatry Rep 2020;22:1.

10. Wu Y-T, Zhang C, Liu H, et al. Perinatal depression of women along with 2019 novel coronavirus breakout in China. Soc Sci Res Netw 2020. Available at: https://papers .ssrn.com/sol3/papers.cfm?abstract_id=3539359 Accessed February 17, 2020.

11. Sun G, Wang F, Cheng Y. Perinatal depression during the COVID-19 epidemic in Wuhan, China. Soc Sci Res Netw. Available at: https://papers.ssrn.com/sol3/papers.cfm?abstract_ id=3576929 Accessed April 11, 2020.

12. Cameron EE, Joyce K, Delaquis CP, Reynolds K, Protudjer J, Roos LE. Maternal psychological distress \& mental health service use during the COVID-19 pandemic. J Affective Disorders 2020;276:765-774.

13. Haight SC, Byatt N, Moore Simas TA, Robbins CL, Ko JY. Recorded diagnoses of depression during delivery hospitalizations in the United States, 20002015. Obstet Gynecol 2019;133:1216-1223.

14. Lancaster CA, Gold KJ, Flynn HA, Yoo H, Marcus SM, Davis MM. Risk factors for depressive symptoms during pregnancy: A systematic review. Am J Obstet Gynecol 2010;202:5-14.

15. Field T. Prenatal depression risk factors, developmental effects and interventions: A review. J Pregnancy Child Health 2017;4:301.
16. Racine N, Zumwalt K, McDonald S, Tough S, Madigan S. Perinatal depression: The role of maternal adverse childhood experiences and social support. J Affect Disord 2020; 263:576-581.

17. Atzl VM, Narayan AJ, Rivera LM, Lieberman AF. Adverse childhood experiences and prenatal mental health: Type of ACEs and age of maltreatment onset. J Fam Psychol 2019; 33:304-314.

18. Molyneaux E, Poston L, Ashurst-Williams S, Howard LM. Obesity and mental disorders during pregnancy and postpartum: A systematic review and meta-analysis. Obstet Gynecol 2014;123:857-867.

19. O’Hara MW, Wisner KL. Perinatal mental illness: Definition, description and aetiology. Best Pract Res Clin Obstet Gynaecol 2014;28:3-12.

20. Mahon PB, Payne JL, MacKinnon DF, et al. Genome-wide linkage and follow-up association study of postpartum mood symptoms. Am J Psychiatry 2009;166:1229-1237.

21. Hutchens BF, Kearney J. Risk factors for postpartum depression: An umbrella review. J Midwifery Womens Health 2020;65:96-108.

22. Hahn-Holbrook J, Cornwell-Hinrichs T, Anaya I. Economic and health predictors of national postpartum depression prevalence: A systematic review, meta-analysis, and meta-regression of 291 studies from 56 countries. Front Psychiatry 2018;8:248.

23. Guintivano J, Manuck T, Meltzer-Brody S. Predictors of postpartum depression: A comprehensive review of the last decade of evidence. Clin Obstet Gynecol 2018;61:591-603.

24. Field T, Diego M, Hernandez-Reif M. Prenatal depression effects on the fetus and newborn: A review. Infant Behav Dev 2006;29:445-455.

25. Slomian J, Honvo G, Emonts $\mathrm{P}$, Reginster J-Y, Bruyère O. Consequences of maternal postpartum depression: A systematic review of maternal and infant outcomes. Womens Health (Lond) 2019;15:1745506519844044.

26. Barr JA. Postpartum depression, delayed maternal adaptation, and mechanical infant caring: A phenomenological hermeneutic study. Int J Nurs Stud 2008;45:362-369.

27. Goodman JH. Perinatal depression and infant mental health. Arch of Psychiatr Nurs 2019;33:217-224.

28. Letourneau NL, Dennis CL, Benzies K, et al. Postpartum depression is a family affair: Addressing the impact on mothers, fathers, and children. Issues Ment Health Nurs 2012;33:445-457.

29. Meltzer-Brody S. New insights into perinatal depression: Pathogenesis and treatment during pregnancy and postpartum. Dialogues Clin Neurosci 2011;13:89-100.

30. Muzik M, Borovska S. Perinatal depression: Implications for child mental health. Ment Health Fam Med 2010;7: 239-247.

31. Cox EQ, Sowa NA, Meltzer-Brody SE, Gaynes BN. The perinatal depression treatment cascade: Baby steps toward improving outcomes. J Clin Psychiatry 2016;77:1189-1200.

32. Drury SS, Scaramella L, Zeanah CH. The neurobiological impact of postpartum maternal depression: Prevention and intervention approaches. Child Adolesc Psychiatr Clin N Am 2016;25:179-200.

33. Dinan TG, Cryan JF. Microbes, immunity, and behavior: Psychoneuroimmunology meets the microbiome. Neuropsychopharmacol 2017;42:178-192.

34. Sanders A, Rackers H, Kimmel M. A role for the microbiome in mother-infant interaction and perinatal depression. Int Rev Psychiatry 2019;31:280-294. 
35. Yonkers KA, Smith MV, Gotman N, Belanger K. Typical somatic symptoms of pregnancy and their impact on a diagnosis of major depressive disorder. Gen Hosp Psychiatry 2009;31:327-333.

36. Horowitz JA, Goodman JH. Identifying and treating postpartum depression. J Obstet Gynecol Neonatal Nurs 2005; 34:264-273.

37. Accortt EE, Wong MS. It is time for routine screening for perinatal mood and anxiety disorders in obstetrics and gynecology settings. Obstet Gynecol Surv 2017;72:553-568.

38. Byatt N, Biebel K, Lundquist RS, et al. Patient, provider, and system-level barriers and facilitators to addressing perinatal depression. J Reprod Infant Psychol 2012;30:436449.

39. American College of Obstetricians and Gynecologists. ACOG Committee Opinion No. 757: Screening for perinatal depression. Obstet Gynecol 2018;132:e208-e212.

40. Siu AL, Bibbins-Domingo K, Grossman DC, et al. Screening for depression in adults: U.S. Preventive Services Task Force Recommendation Statement. JAMA 2016;315:380-387.

41. Sidebottom AC, Harrison PA, Godecker A, Kim H. Validation of the Patient Health Questionnaire (PHQ)-9 for prenatal depression screening. Arch Womens Ment Health 2012;15:367-374.

42. Sockol LE, Epperson CN, Barber JP. A meta-analysis of treatments for perinatal depression. Clin Psychol Rev 2011; 31:839-849.

43. Sockol LE. A systematic review and meta-analysis of interpersonal psychotherapy for perinatal women. J Affect Disord 2018;232:316-328.

44. Sockol LE. A systematic review of the efficacy of cognitive behavioral therapy for treating and preventing perinatal depression. J Affect Disord 2015;177:7-21.

45. Lusskin SI, Khan SJ, Ernst C, Habib S, Fersh ME, Albertini ES. Pharmacotherapy for perinatal depression. Clin Obstet Gynecol 2018;61:544-561.

46. Adams J, Lui CW, Sibbritt D, et al. Women's use of complementary and alternative medicine during pregnancy: A critical review of the literature. Birth 2009;36:237-245.

47. Hall HG, Griffiths DL, McKenna LG. The use of complementary and alternative medicine by pregnant women: A literature review. Midwifery 2011;27:817-824.

48. Eustis EH, Ernst S, Sutton K, Battle CL. Innovations in the treatment of perinatal depression: The role of yoga and physical activity interventions during pregnancy and postpartum. Curr Psychiatry Rep 2019;21:133.

49. Deligiannidis KM, Freeman MP. Complementary and alternative medicine therapies for perinatal depression. Best Pract Res Clin Obstet Gynaecol 2014;28:85-95.

50. Matthews J, Huberty JL, Leiferman JA, McClain D, Larkey LK. Perceptions, uses of, and interests in complementary health care approaches in depressed pregnant women: The PAW Survey. J Evid Based Complementary Altern Med 2017;22:81-95.

51. Mills JL. Strategies for preventing folate-related neural tube defects: Supplements, fortified foods, or both? JAMA 2017;317:144-145.

52. Sheffield KM, Woods-Giscombé CL. Efficacy, feasibility, and acceptability of perinatal yoga on women's mental health and well-being: A systematic literature review. J Holist Nurs 2016;34:64-79.
53. Luberto CM, Park ER, Goodman JH. Postpartum outcomes and formal mindfulness practice in mindfulness-based cognitive therapy for perinatal women. Mindfulness (N Y) 2018;9:850-859.

54. Dimidjian S, Goodman SH, Felder JN, Gallop R, Brown AP, Beck A. Staying well during pregnancy and the postpartum: A pilot randomized trial of mindfulness-based cognitive therapy for the prevention of depressive relapse/recurrence. J Consult Clin Psychol 2016;84:134-145.

55. Reza N, Deligiannidis KM, Eustis EH, Battle CL. Complementary health practices for treating perinatal depression. Obstet Gynecol Clin North Am 2018;45:441-454.

56. Uebelacker LA, Battle CL, Sutton KA, Magee SR, Miller IW. A pilot randomized controlled trial comparing prenatal yoga to perinatal health education for antenatal depression. Arch Womens Ment Health 2016;19:543-547.

57. Kozhimannil KB, Adams AS, Soumerai SB, Busch AB, Huskamp HA. New Jersey's efforts to improve postpartum depression care did not change treatment patterns for women on Medicaid. Health Aff (Millwood) 2011;30:293301.

58. Rhodes AM, Segre LS. Perinatal depression: A review of U.S. legislation and law. Arch Womens Ment Health 2013; 16:259-270.

59. Signed MA, March IC. MOTHERS Act Signed into Law. 2010. Available at: https://core.ac.uk/download/pdf/11353591 .pdf Accessed October 30, 2020.

60. Aitken Z, Garrett CC, Hewitt B, Keogh L, Hocking JS, Kavanagh AM. The maternal health outcomes of paid maternity leave: A systematic review. Soc Sci Med 2015; 130:32-41.

61. International Labour Organization. Maternity and paternity at work: Law and practice across the world. 2014. Available at: https://www.ilo.org/wcmsp5/groups/public/—dgreports/— dcomm/—publ/documents/publication/wcms_242615.pdf Accessed April 3, 2020.

62. National Partnership for Women and Families. State Paid Family and Medical Leave Insurance Laws. 2019. Available at: https://www.nationalpartnership.org/our-work/resources/ economic-justice/paid-leave/state-paid-family-leave-laws.pdf Accessed April 3, 2020.

63. Rossin-Slater M, Ruhm CJ, Waldfogel J. The effects of California's paid family leave program on mothers' leavetaking and subsequent labor market outcomes. J Policy Anal Manage 2013;32:224-245.

64. Bartel A, Doran E, Ruhm C, Waldfogel J. Panel Paper: The effects of California's paid family leave law on maternal psychological health. 2019. Available at: https://appam .confex.com/appam/2019/webprogram/Paper32688.html Accessed April 3, 2020.

Address correspondence to: Rada K. Dagher, PhD, MPH Division of Scientific Programs, National Institute on Minority Health and Health Disparities

National Institutes of Health

6707 Democracy Boulevard, Suite 800 Bethesda, MD 20892

USA

E-mail: rada.dagher@nih.gov 